How to Cite

Aryani, L. N. A., \& Lesmana, C. B. J. (2019). Neuropsychiatric factor and polymorphism gene in internet addiction. International

Journal of Health \& Medical Sciences, 2(1), 39-44. https://doi.org/10.31295/ijhms.v2n1.90

\title{
Neuropsychiatric Factor and Polymorphism Gene in Internet Addiction
}

\author{
Luh Nyoman Alit Aryani \\ Udayana University, Denpasar, Indonesia \\ Corresponding author email: alitaryani@ rocketmail.com \\ Cokorda Bagus Jaya Lesmana \\ Udayana University, Denpasar, Indonesia
}

\begin{abstract}
Internet addiction (IA) has been described as 'excessive or poorly controlled preoccupation, urges, or behaviors regarding computer use and internet access that lead to impairment or distress. Excessive internet user has emerged as a leading cause of behavioral and developmental problems in adolescents. There are several factors that influence the occurrence of internet addiction. The risk factors can be categorized by a social factor, psychiatric factor and biological factor. Some studies have found varied results. Molecular and functional imaging techniques have been increasingly used for the analysis of neurobiological changes and neurochemical correlates of IA. MRI (Magnetic Resonance Imaging) studies demonstrate that structural changes in the frontal cortex are associated with functional abnormalities in Internet-addicted subjects. Recent research has implicated the role of the striatal dopaminergic system in the behavioral maladaptation associated with IA. It provided evidence for a molecular genetic link between serotonergic and dopaminergic neurotransmission and Internet addiction like dopamine polymorphism the DRD2/ANKKI Taq Ia and COMT Val158metpolymorphism and the gene coding for the nicotinic acetylcholine receptor subunit alpha 4 (CHRNA4). As both the genetic and personality traits are known to play a role in other addiction it is likely that same mechanism (biopsychosocial) underlie Internet addiction.

Keywords---internet addiction, neuropsychiatric factor, polymorphism gene.
\end{abstract}

\section{Introduction}

Many psychiatric disorders that arise due to internet addiction. Internet addiction is an emerging public health issue. The rise of new technologies, such as the Internet and associated social media sites, has exposed adolescents to online risks (pornography exposure, cyberbullying and Internet addiction) and health risks (depression and suicide), which have increased the rates of youth morbidity and mortality (Michelle et al., 2017).

There are some psychiatric disorders that are identified because of internet addiction. Internet Addiction (IA) is a growing problem, particularly among young people. Mental health in adolescents, such as Attention Deficit disorder, obsessive-compulsive disorder, depression, anxiety, and hostility, has been reported as comorbidities of Internet Addiction (Lam, 2018). Addiction to substances or activities can profoundly affect people's health and sometimes lead to serious social problems (Zhu et al., 2015). Internet addiction specifically refers to the following feelings and attitudes: the preference of the virtual life over real life, and the use of the internet to escape from negative emotions and thought. The excessive use of the internet can easily lead to compulsive if some predisposing personal factors are present, such as temperament (Abdullah, 2017; Espinosa et al., 2018; Praharsini et al., 2018).

Behavioral addictions are complex disorders with interacting factors, including environmental factors, comorbidity, personality traits, and stress responsivity. Behavioral addictions involve dysfunction in several brain regions, principally the frontal cortex and limbic system and disruption in neurobiological processes underlying both sensitivity to reward and inhibitory control can lead to compulsive behaviors (Fattore et al., 2014).

Definition of "behavioural addictions" has been recently expanded to encompass any behaviour characterized by (i) a feeling of tension or arousal before the action, (ii) gratification and/or relief at the time of executing the act, (iii)

ISSN 2632-9433

Received Jan 18, 2019 / Accepted Jun 27, 2019 / Published Jul 31, 2019 
an inability to resist an urge or drive even against great obstacles or dangers, and (iv) the absence of consideration for the negative consequences that may affect family, friends, or work. As such, behavioral addictions include compulsive food intake and sexual activity, pathological gambling and Internet addiction, excessive exercising, compulsive (Fattore et al., 2014; Jain, 2017; Suryasa et al., 2018).

\section{Psychiatric Factor related to Internet Addiction}

Parenting is very important in this disorder. In previous studies, had found that parental mental health was related to their children's IA and also a parent and adolescent IA relationship. To further explore the complex relationships among parent and child mental health, parental IA and adolescent IA (Lam, 2018). The other study indicates a high prevalence of internet addiction among Chinese adolescent internet users, advances our understanding of the association of IA with stressful life events and psychological symptoms and provides evidence for the prevention of IA (Jie et al., 2014). Affective and anxiety disorders have been linked to IA. IA appears to be associated with mental disorders in adolescents and adults, and with developmental traits. Comorbid symptoms of anxiety and IA have been found in adolescents and adults. Additionally, increased stress levels in adults and stressful life events in adolescents have been associated with IA. A study of college students found strong positive correlations between stress, anxiety, depression, and IA. Thus, IA may be associated with increased levels of psychological distress covering a range of psychological conditions (Michelle et al., 2017).

One-seventh of the junior high school students canvassed in one study were addicted to the Internet. Adolescents with an Internet addiction had greater levels of online risks (pornography/violence exposure, cyberbullying, and online sexual solicitation) and health risks (less vegetable/fruit intake, more consumption of sugary drinks, smoking, consumption of alcohol, low self-esteem, and depression) (Chang et al., 2015; Bento, 2018).

Early puberty was positively associated with Internet use in junior high school students. Particularly, early maturing adolescent boys had a higher likelihood of online pornography viewing. Given the potentially negative impacts of pornography exposure on subsequent sexual behaviors, appropriate health education should be provided to those who are at high risk. When weight status and pubertal timing were considered, sexual maturation came in as an important confounder in the link between the purposes of Internet use and weight status. Meanwhile, the gender difference was noted in the association between adolescent weight status and Internet use (Tsai et al., 2018). The causal relationship between Internet addiction and depression varies by gender. Male adolescents were more likely to overindulge on the Internet because of depression. Female adolescents were more prone to depression as a result of Internet addiction (Tsai et al., 2018; Liang et al., 2016).

\section{Neurobiology factor related to Internet Addiction}

Some neurobiological studies have varied results. From a study obtained nuclear imaging findings indicate that IA is associated with dysfunction of the brain dopaminergic systems. Abnormal dopamine regulation of the prefrontal cortex (PFC) could underlie the enhanced motivational value and uncontrolled behavior over Internet overuse in addicted subjects (Zhu et al., 2015).

Accumulating evidence in the field of neurobiological studies is demonstrating that prefrontal cortex (PFC) plays a significant role in the development of IA. This notion is suggesting a particular liability of adolescents, due to their incomplete, yet fast-developing maturation of that specific area of the brain. The cortical thickness within the orbital PFC was found to be reduced in adolescents suffering of Internet gaming, suggesting them to possess a somewhat less mature cognitive mechanism for efficient inhibitory control (Cerniglia et al., 2016).

It is known that addictions activate a combination of sites in the brain associated with pleasure, known together as the "reward center" or "pleasure pathway" of the brain. When activated, dopamine release is increased, along with opiates and other neurochemicals. Over time, the associated receptors may be affected, producing tolerance or the need for increased stimulation of the reward center to produce a "high" and the subsequent characteristic behavior patterns needed to avoid withdrawal. Internet use may also lead specifically to dopamine release in the nucleus accumbens, one of the reward structures of the brain specifically involved in other addictions (Cash et al., 2012).

Early reward-centric models focused on pleasurable aspects of taking drugs and proposed that drugs may "caused" brain circuits involved in responses to "natural" rewards like sex or food. A central component in this circuitry is the nucleus accumbens located in the ventral striatum and receiving dopaminergic innervation from the ventral tegmental area (termed the mesolimbic dopamine system). This nucleus accumbens has at times been termed the brain's "reward center," given that all known drugs with abuse potential, as well as natural rewards, lead to dopamine release in this structure. However, a broader range of neurotransmitters 
(including opioids, cannabinoids, serotonin, norepinephrine, acetylcholine, glutamate, and $\gamma$-aminobutyric acid, contributes to addiction, and molecular entities (receptors, transporters) for sensing these neurotransmitters are expressed in specific brain regions (Potenza, 2013).

The other study demonstrates decreased rsFC (Frontal Cortex) strength between the VTA (Ventral Tegmental Area) and NAcc (Nucleus Accumben) among individuals with IGD (Internet Gaming Disorder). The study report associations between VTA and NAcc connectivity and self-reported craving among individuals with IGD. The study results in parallel findings from substance addictions, suggesting possibly shared neurobiological substrates between IGD and substance addictions and providing additional support to the notion that IGD is a behavioral addiction (Zhang \& Ma, 2015).

From MRI studies and EEG (Electro Encephalo Graphy) were conducted that found that those subject classified as internet addict had lower brain than the normal group. This effect was different for the even related potential component N2 and for the P3 amplitudes. Studies investigating brain gray matter density (GMD) demonstrated changed in an adolescent with internet addiction using voxel-based morphometry (VBM) compared with healthy controls. Internet-addicted adolescent had lower GMD in the left anterior cingulate cortex, left insula and left lingual gyrus. The brain regions showing structural changes in IA are known to be involved in reward, emotion generation and processing, executive attention, decision making, and cognitive control. The pattern of IA-related structural abnormalities in the brain is also shown to be similar, to some extent, to that observed in substance addiction (Montag, 2017). The ventral striatum and pre-frontal brain areas are considered important neural contributors to the interaction of cue-reactivity and craving with reduced executive functions and diminished decision-making skills in individuals with specific Internet-use disorders (Brand et al., 2016).

\section{Polymorphism Gene in Internet Addiction}

Currently, there are several studies that look at gene relations with internet addiction. In individuals who are vulnerable to addiction, repetitive exposure to the agent induces long-lasting neuroadaptive changes that further promote drug-seeking behaviors and ultimately lead to persistent and uncontrolled patterns of use that constitute addiction. These neuroadaptive changes are the bases for tolerance, craving, and withdrawal and lead to a motivational shift. Evidence from family, adoption, and twin studies converges on the relevance of genetic factors in the development of addictions including SUD (Substance Use Disorder) and gambling. The identification of specific genes and functional loci moderating vulnerability has been challenging because of the genetic complexity of addictive disorders. This complexity derives from multiple sources including incomplete penetrance, phenocopies, variable expressivity, gene-environment interactions, genetic heterogeneity, polygenicity, and epistasis (Ducci \& Goldman, 2012).

Genetic polymorphism is defined as the inheritance of a trait controlled by a single genetic locus with two alleles, in which the least common allele has a frequency of about $1 \%$ or greater. Genetic polymorphism is a difference in DNA (deoxyribonucleic acid) sequence among individuals, groups, or populations. Sources include single nucleotide polymorphisms (SNPs), sequence repeats, insertions, deletions, and recombination. Genetic polymorphisms may be the result of chance processes or may have been induced by external agents such as viruses or radiation. If a difference in DNA sequence among individuals has been shown to be associated with the disease, it will usually be called a genetic mutation. Changes in the DNA sequence that have been confirmed to be caused by external agents are also generally called 'mutations' rather than 'polymorphisms'. SNPs are the most common type of genetic variations in humans. Understanding the functions of SNPs can greatly help us understand the genetics of human phenotype variation, especially the genetic basis of complex human diseases. DNA polymorphisms comprise three types: tandem repeat polymorphism, copy-number variations, and SNPs. Detection of these polymorphisms can be carried out by different methods including allele-specific PCR, restriction fragment length polymorphism, the microarray technique, and whole-genome sequencing (Somaia \& Mona, 2012).

The findings of the other study demonstrated that the overall use of the Internet is greatly affected by one's ability to sustain attention. Carriers of the alleles associated with decreased attentiveness (DRD4 2R and 7R, 5HTTLPR long) are protected from Internet overuse (Sun \& Spathis, 2016).

The transgenerational inheritance of epigenetic traits, together with the neurobehavioral adaptions to previous environmental stimuli, serves as an important mechanism during evolution. The epigenome lasts for only several generations, and the rapid removal of such "memory" allows further "writing" of new environmental conditions. The epigenome examinations also permit the prediction and potential therapy against certain birth defects resulted from environmental toxin/stress (Yeong et al., 2006).

Several studies from Asia provided first evidence for a molecular genetic link between serotonergic and dopaminergic neurotransmission and Internet addiction. The present report offers data on a new candidate gene in the 
investigation of Internet addiction the gene coding for the nicotinic acetylcholine receptor subunit alpha 4 (CHRNA4). The T- variant (CC genotype) of the rs1044396 polymorphism on the CHRNA4 gene occurred significantly more frequently in the subject (Montag \& Kirsch, 2012).

The other study suggests that male adolescents with internet addiction tendency have the specific temperament and genetic polymorphisms like chemical dependence (Montag, 2017). One study used reward dependence scale in Cloninger Temperament and Character Inventory and frequencies of two dopamine polymorphism the DRD2/ANKKI Taq Ia and COMT Val158metpolymorphism. Results of the study found that the 132 problem Internet users showed higher elevation of the CC genotype of rs 044396 (genetic variation of nicotinic acetylcholine receptor gene) compared to controls. These neurotransmitters play a significant role in activating the brains reward system (Montag \& Kirsch, 2012).

A study also identified the influence of the gene coding for the alpha4 subunit of the nicotinic acetylcholine receptor (CHRNA4) on IGD (Internet Gaming Disorder). With respect to addiction, a biological link between CHRNA4 and the nicotine-addiction phenotype has been established. One possible explanation for the effect of the CHRNA4 gene on addiction is that the cholinergic pathway is involved in actions in the mesolimbic dopaminergic system, which serves a principal role in the acquisition of addictive behavior. Furthermore, previous studies have shown a significant association between polymorphisms in CHRNA4 and the psychological risk attitude, response inhibition, and harm avoidance. Impaired response inhibition is an important issue in behavioral addiction. These combined results suggest that the nicotinic acetylcholine receptor may play a role in IGD by modulating the dopaminergic pathway and may affect the addiction phenotype, such as response inhibition (Jeong et al., 2017).

\section{Summary}

Behavioral addictions are complex disorders with interacting factors, including environmental factors, comorbidity, personality traits, and stress responsivity. IA appears to be associated with mental disorders in adolescents and adults with comorbid symptoms of anxiety and depression. Abnormal dopamine regulation of the prefrontal cortex (PFC) could underlie the enhanced motivational value and uncontrolled behavior over Internet overuse in addicted subjects. The present studies report offers data on a new candidate gene in the investigation of Internet addiction the gene coding for the nicotinic acetylcholine receptor subunit alpha 4 (CHRNA4) and provided evidence for a molecular genetic link between serotonergic and dopaminergic neurotransmission and Internet addiction. The result of the other study showed that male adolescents with internet addiction tendency have specific temperament and genetic polymorphisms like chemical dependence. Emerging evidence has shown that changes in brain structure and activity related to IA are relevant to brain regions involved in reward, motivation, and memory, as well as cognitive control. IA has become a serious problem worldwide, a need for effective treatment is becoming increasingly urgent. Both psychiatric and biologic approaches have been applied to treat IA. The polygenic nature of addiction has implications for the manner in which genetic predictors may eventually be used in the treatment and genetic counseling.

\section{Acknowledgments}

The authors would like to express gratitude to DR dr. Cok Bagus Jaya Lesmana SpKJ (K), MARS. Department of Psychiatric, Faculty of Medicine Udayana University, Denpasar, Indonesia, for reviewing this manuscript.

\section{References}

Abdullah, M. (2017). The Relationship between Internet Addiction and Temperament among Children and Adolescents. Psychology and Behavioral Science International Journal, 5(5).

Bento, A. C. (2018). Internet of Things: An Experiment with Residential Automation for Robotics Classes. International Research Journal of Management, IT and Social Sciences, 5(2), 113-119. https://doi.org/10.21744/irjmis.v5n2.51

Brand, M., Young, K. S., Laier, C., Wölfling, K., \& Potenza, M. N. (2016). Integrating psychological and neurobiological considerations regarding the development and maintenance of specific Internet-use disorders: An Interaction of Person-Affect-Cognition-Execution (I-PACE) model. Neuroscience \& Biobehavioral Reviews, 71, 252-266. https://doi.org/10.1016/j.neubiorev.2016.08.033

Cash, H., D Rae, C., H Steel, A., \& Winkler, A. (2012). Internet addiction: A brief summary of research and practice. Current psychiatry reviews, 8(4), 292-298. https://doi.org/10.2174/157340012803520513

Cerniglia, L., Zoratto, F., Cimino, S., Laviola, G., Ammaniti, M., \& Adriani, W. (2017). Internet Addiction in adolescence: Neurobiological, psychosocial and clinical issues. Neuroscience \& Biobehavioral Reviews, 76, 174- 
184. https://doi.org/10.1016/j.neubiorev.2016.12.024

Chang, F. C., Chiu, C. H., Miao, N. F., Chen, P. H., Lee, C. M., Chiang, J. T., \& Pan, Y. C. (2015). The relationship between parental mediation and Internet addiction among adolescents, and the association with cyberbullying and depression. Comprehensive Psychiatry, 57, 21-28. https://doi.org/10.1016/j.comppsych.2014.11.013

Ducci, F., \& Goldman, D. (2012). The genetic basis of addictive disorders. Psychiatric Clinics, 35(2), $495-519$. https://doi.org/10.1016/j.psc.2012.03.010

Espinosa, A. H. R., Estevez, A. G., Roche, J. R. F., Figarola, Y. M., \& Rodriguez, D. L. (2018). Psychological intervention for development of disease awareness in addicts: Villa Colibri therapeutic community of Santiago de Cuba. International Journal of Health Sciences, 2(3), 72-80. https://doi.org/10.29332/ijhs.v2n3.232

Fattore, L., Melis, M., Fadda, P., \& Fratta, W. (2014). Sex differences in addictive disorders. Frontiers in neuroendocrinology, 35(3), 272-284. https://doi.org/10.1016/j.yfrne.2014.04.003

Ismail, S., \& Essawi, M. (2012). Genetic polymorphism studies in humans. Middle East Journal of Medical Genetics, 1(2), 57-63. https://doi.org/10.1097/01.MXE.0000415225.85003.47

Jain, P. (2017). Effect of online education trend on quality management. International Journal of Health Sciences, 1(1), 1-5. https://doi.org/10.21744/ijhs.v1i1.16

Jeong, J. E., Rhee, J. K., Kim, T. M., Kwak, S. M., Bang, S. H., Cho, H., ... \& Choi, J. S. (2017). The association between the nicotinic acetylcholine receptor $\alpha 4$ subunit gene (CHRNA4) rs1044396 and Internet gaming disorder in Korean male adults. PloS one, 12(12), e0188358. https://doi.org/10.1371/journal.pone.0188358

Kim, E. Y., Lee, Y. S., Han, D. H., Suh, D. S., \& Kee, B. S. (2006). Temperament and Genetic Polymorphism in Korean Male Adolescents with Internet Addiction Tendency. Journal of Korean Neuropsychiatric Association, 45(5), 468-475.

Liang, L., Zhou, D., Yuan, C., Shao, A., \& Bian, Y. (2016). Gender differences in the relationship between internet addiction and depression: A cross-lagged study in Chinese adolescents. Computers in Human Behavior, 63, 463470. https://doi.org/10.1016/j.chb.2016.04.043

Lozano, R., Fullman, N., Abate, D., Abay, S. M., Abbafati, C., Abbasi, N., ... \& Abdel-Rahman, O. (2018). Measuring progress from 1990 to 2017 and projecting attainment to 2030 of the health-related Sustainable Development Goals for 195 countries and territories: a systematic analysis for the Global Burden of Disease Study 2017. The Lancet, 392(10159), 2091-2138.

McNicol, M. L., \& Thorsteinsson, E. B. (2017). Internet addiction, psychological distress, and coping responses among adolescents and adults. Cyberpsychology, Behavior, and Social Networking, 20(5), 296-304. https://doi.org/10.1089/cyber.2016.0669

Montag, C., \& Reuter, M. (2017). Internet Addiction (pp. 143-150). Springer International Publishing.

Montag, C., Kirsch, P., Sauer, C., Markett, S., \& Reuter, M. (2012). The role of the CHRNA4 gene in Internet addiction: a case-control study. Journal of addiction medicine, 6(3), 191-195. https://doi.org/10.1097/ADM.0b013e31825ba7e7

Potenza, M. N. (2013). Biological contributions to addictions in adolescents and adults: Prevention, treatment, and policy implications. Journal of Adolescent Health, 52(2), https://doi.org/10.1016/j.jadohealth.2012.05.007

Praharsini, I., Suryawati, N., Indira, I. E., \& Sanjiwani, S. P. (2018). High level of tumor necrosis alpha and serum interferon gamma as risk factors for progression of vitiligo disease. International Journal of Health Sciences, 2(2), 1-8. https://doi.org/10.29332/ijhs.v2n2.109

Sun, C., Spathis, R., Lum, J. K., Sankaranarayanan, K., \& Chan, C. W. (2016). Genetic-linked Inattentiveness Protects Individuals from Internet Overuse: A Genetic Study of Internet Overuse Evaluating Hypotheses Based on Addiction, Inattention, Novelty-seeking and Harm-avoidance. Informing Science, 19.

Suryasa, I. W., Prayoga, I. G. P. A., \& Werdistira, I. W. A. (2018). Attitudes toward the use of internet for students. International Journal of Physical Sciences and Engineering, 2(2), 32-38. https://doi.org/10.29332/ijpse.v2n2.141

Tang, J., Yu, Y., Du, Y., Ma, Y., Zhang, D., \& Wang, J. (2014). Prevalence of internet addiction and its association with stressful life events and psychological symptoms among adolescent internet users. Addictive Behaviors, 39(3), 744-747. https://doi.org/10.1016/j.addbeh.2013.12.010

Tsai, M. C., Strong, C., Chen, W. T., Lee, C. T., \& Lin, C. Y. (2018). Longitudinal impacts of pubertal timing and weight status on adolescent Internet use: Analysis from a cohort study of Taiwanese youths. PloS one, 13(5), e0197860. https://doi.org/10.1371/journal.pone.0197860

Zhang, J. T., Ma, S. S., Yip, S. W., Wang, L. J., Chen, C., Yan, C. G., ... \& Fang, X. Y. (2015). Decreased functional connectivity between ventral tegmental area and nucleus accumbens in Internet gaming disorder: evidence from resting state functional magnetic resonance imaging. Behavioral and Brain Functions, 11(1), 37. 
Zhu, Y., Zhang, H., \& Tian, M. (2015). Molecular and functional imaging of internet addiction. BioMed research international, 2015. 